\title{
Thoracic endovascular repair for acute type A aortic dissection: operative technique
}

\author{
Aamir Shah, Ali Khoynezhad \\ Division of Cardio-Thoracic Surgery, Cedars-Sinai Medical Center, Los Angeles, CA, USA \\ Correspondence to: Ali Khoynezhad, MD, PhD. Division of Cardio-Thoracic Surgery, Cedars-Sinai Medical Center, 8700 Beverly Blvd, Los Angeles, \\ CA 90048, USA. Email: Ali.khoynezhad@cshs.org.
}

\begin{abstract}
Acute type A aortic dissection is a potentially lethal condition which requires immediate diagnostic and therapeutic intervention. Open surgical repair remains the standard of care as survival rates continue to improve in the modern surgical era. Unfortunately, up to twenty percent of patients are denied surgical therapy because they are deemed medically unfit to undergo open repair. The application of thoracic endovascular aortic repair (TEVAR) has changed the treatment paradigm for aortic disease involving the descending thoracic aorta and may be a viable rescue option for patients with type A dissection who are not eligible for open surgical repair. New endovascular devices and advanced image-guided procedures are continually evolving. This article summarizes the pathology of aortic dissection and focuses on currently available endovascular solutions for transapical and transfemoral stent graft deployment for acute aortic dissection involving the ascending aorta for selected patients who are ineligible for open surgical repair.
\end{abstract}

Keywords: Aortic dissection; ascending aorta; stent graft; endovascular

Submitted Jun 14, 2016. Accepted for publication Jun 22, 2016.

doi: 10.21037/acs.2016.07.08

View this article at: http://dx.doi.org/10.21037/acs.2016.07.08

\section{Introduction}

Acute aortic dissection is a catastrophic condition which arises from a tear in the intima of the aorta, resulting in separation between the intimal and medial layers of the aortic wall and subsequent entry of blood flow within the media. Extension of the dissection may be antegrade, retrograde or bidirectional. The pressurization of the false lumen of the aorta can lead to coronary, brachiocephalic or branch vessel malperfusion, as well as aortic valve insufficiency, or aortic rupture. The incidence of aortic dissection is approximately 2.0 to 3.5 per 100,000 people per year (1-3). The most common risk factors are hypertension, connective tissue disorders and trauma (4).

The most recent data collected from the International Registry of Acute Aortic Dissection (IRAD) indicate that $67 \%$ of the patients with acute aortic dissection presented with type A dissection (5). From 1996 to 2013, the percentage of patients undergoing surgical treatment for acute type A dissection in the IRAD Registry increased from $79 \%$ to $90 \%$ and the overall surgical mortality improved from $25 \%$ to $18 \%$. However, the in-hospital mortality of patients unfit for surgical repair remained unchanged at approximately $60 \%$ (5). This underscores the value of endovascular therapy as a possible treatment alternative in the $10-20 \%$ of patients with type A dissection who are medically unfit to undergo open surgical repair.

The application of thoracic endovascular aortic repair (TEVAR) has dramatically changed the treatment paradigm for aortic disease of the thoracic aorta. TEVAR is typically better tolerated by a more elderly and unwell patient cohort due to less invasive nature of stent grafting, which obviates the need for thoracotomy, cardiopulmonary bypass and deep hypothermic circulatory arrest. In this article we describe the technique of how to perform TEVAR in the ascending aorta for acute type A aortic dissection in patients with anatomic suitability who are medically unfit for surgical repair.

The entry tear in aortic dissection is located in the ascending aorta in two third of cases and more than half of these are within the first $2 \mathrm{~cm}$ of the ascending aorta (6). 


\begin{tabular}{l} 
Table 1 Anatomical requirements for ascending aortic TEVAR \\
\hline Proximal and distal landing zones \\
Length $>10 \mathrm{~mm}$ \\
Diameter $>16$ and $<42 \mathrm{~mm}$ \\
No significant difference between proximal and distal landing \\
zone $(<10 \%)$ \\
Absence of calcification or thrombotic material \\
Aortic dissection \\
Intimal tear $>10$ mm above the sinotubular junction \\
Intimal tear $>5 \mathrm{~mm}$ proximal to the innominate artery \\
No aortic regurgitation \\
Access vessels \\
Diameter of the common and external iliac artery $>7 \mathrm{~mm}$ \\
\hline TEVAR, thoracic endovascular aortic repair.
\end{tabular}

The next most common position of the primary tear is the isthmus of the aorta, just beyond the ligamentum arteriosum. Re-entry tears through which blood can communicate between a true and false lumen are often at the ostia of branch vessels.

\section{Classification}

The three major classification systems for aortic dissections are The DeBakey, Stanford and more recent DISSECT classification. The DeBakey classification describes which part of the aorta is involved in the dissection: in type I dissection $(60 \%)$ both ascending and descending thoracic aorta are involved, in type II only the ascending part is involved (10-15\%), and in type III only descending aorta is involved (25-30\%) (7). The Stanford classification categorises aortic dissections according to whether the ascending aorta is involved (Stanford type A) or not (Stanford type B). A more recent classification system DISSECT was introduced by Dake in 2013 and is particularly useful for endovascular therapy (8). It encompasses five features: duration, intimal tear location, size of aorta, segmental extent of dissection involvement, clinical complications (malperfusion or rupture) and thrombosis of the false lumen.

\section{Indications and contraindications}

Open repair with replacement of the diseased aorta remains standard therapy for the majority of patients. The primary goals of surgical therapy are to resect the origin of the intimal tear and to reapproximate the intima to the media and adventitia in order to obliterate the false lumen. TEVAR may be considered as an alternative in high-risk or inoperable patients. The goals of TEVAR are similar: to cover the origin of the intimal tear in order to prevent aortic rupture as well as to reduce pressure and promote thrombosis of the false lumen. TEVAR is contraindicated if there is severe aortic valve regurgitation, if the dissection involves the aortic root or if the patient has a connective tissue disorder except as a temporizing solution until definitive surgery can be performed. Table 1 provides a list of anatomic requirements for ascending TEVAR. The origin of the intimal tear should be greater than $10 \mathrm{~mm}$ distal to the sinotubular junction and the proximal and distal landing zones should be greater than $10 \mathrm{~mm}$ in length. The distal landing zone can be extended by performing a left carotid to right carotid artery bypass if coverage of the innominate artery is required to achieve adequate distal seal.

The anatomy and access to the ascending aorta pose significantly greater challenges in comparison with the descending thoracic or abdominal aorta. Guidewires and sheaths must traverse the aortic arch along the ostia of the supra-aortic branches to access the ascending aorta. In a DeBakey type I dissection, this maneuver may potentially be associated with neuro-embolization of thrombus or extension of the dissection.

In addition, the landing zones are angulated and limited in length, bordered distally by the innominate artery and proximally by the sinotubular junction with the coronary arteries and aortic valve just below. There may be significant discrepancies between the sizes of the proximal and distal seal zones. In these cases, carefully imaged and properly planned stent-tapered configurations or custommade devices will address an adequate stent graft alignment over the whole length of the aorta. Injury of the aortic wall in this part of aorta may have fatal consequences such as a retrograde type A aortic dissection (RAAD) or aortic rupture. The incidence of RAAD following TEVAR has been reported to be between $1.3 \%$ and $6.8 \%$ (9-11).

Finally, one must consider the hemodynamic forces to which the ascending aorta is subjected, including significant changes in diameter and area throughout the cardiac cycle. Using dynamic ECG-gated CTA to evaluate pulsatility of the ascending aorta, van Prehn and associates found that the greatest changes in diameter during the cardiac cycle occur $5 \mathrm{~mm}$ distal to the coronary arteries, with a mean maximum diameter change of $17 \%$ (12). The mean change in diameter $5 \mathrm{~mm}$ proximal to the innominate artery was $14 \%$. 


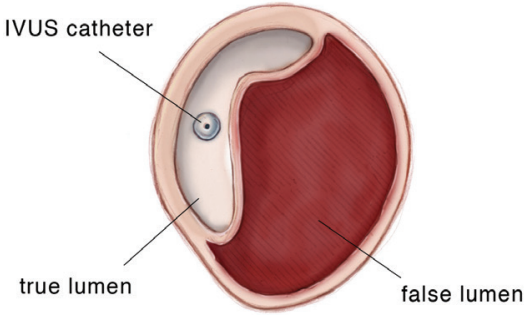

Figure 1 Intravascular ultrasound (IVUS) demonstrating acute aortic dissection with compression of the aortic true lumen by the false lumen. The IVUS catheter is in the false lumen.

These findings must be considered in the design of ascending aortic and branched or fenestrated devices in order to achieve adequate proximal and distal seal as well as device durability. The authors are currently involved with the first FDA-approved investigational device exemption (IDE) for type A aortic dissection, ascending aortic ulcers and intramural hematomas. This IDE excludes any dissections or pathologies involving the aortic root proximal to the sinotubular junction.

\section{Preoperative and adjunctive imaging and monitoring}

Preoperative imaging of the aorta is central to planning the procedure. Gated contrast-enhanced CT scans or noncontrast time-of-flight MRI provides detailed information about the aortic anatomy including the size and quality of the aortic wall, the presence of atherosclerotic plaques or penetrating atherosclerotic ulcers. Furthermore, it allows assessment of proximal and distal landing zones, the size and angulation of access vessels and also demonstrates the location of vital side branches.

Another improvement in imaging technology in complex branched and fenestrated endovascular stent graft cases is the integration of the preoperative CT scan into the operative fluoroscopic image. The stent graft can be positioned in relation to critical branches more accurately and with reduced radiation and contrast medium exposure (13). Fusion images provide the surgeon with precise intraoperative roadmaps for aortic procedures, generated using preoperative images from PET or CTA or MRA scans and overlapped with live intraoperative fluoroscopy images.

Transesophageal echocardiography (TEE) is critical for evaluating cardiac and valve function before and after

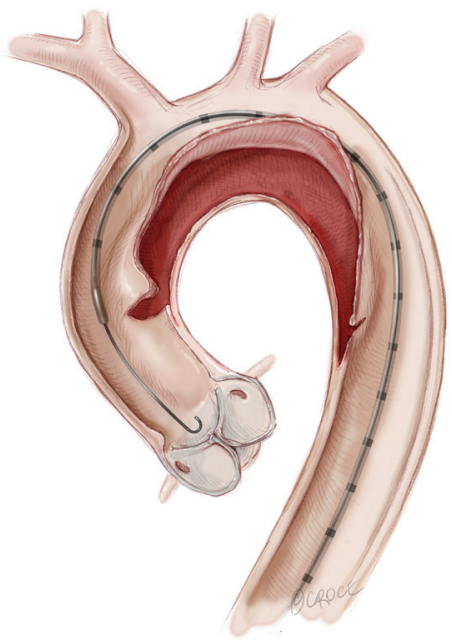

Figure 2 Transfemoral intravascular ultrasound (IVUS) catheter in the ascending aorta.

the procedure. It can also detect complications following stent graft deployment including iatrogenic aortic valve regurgitation or regional wall motion abnormalities because of coronary artery obstruction. Furthermore, TEE may help guide the optimal position of the transapical access as well as confirm true lumen access of the guidewire.

Neurophysiologic monitoring is another adjunct to reduce neurological complications. This is especially relevant to TEVAR in the ascending aorta due to the proximity of the supra-aortic vessels and their vulnerability to embolization, coverage or dissection. To ensure the integrity of the supra-aortic vessels and adequate cerebral perfusion, several tools may be used. Transcutaneous nearinfrared spectroscopy and transcranial cerebral oximetry both provide continuous real-time information about cerebral perfusion and oxygenation. Transcranial Doppler (TCD) measures the velocity of blood flow in the cerebral vessels and can detect cerebral micro-emboli and changes in blood flow which may accurately indicate partial or complete coverage of the cerebral vessels.

Intravascular ultrasound (IVUS) is a valuable resource in providing supplemental endoluminal information (see Figures 1,2 ). It is the most accurate modality in measuring the luminal diameter of vessels. This technology can also be used to identify the position of branch vessels, inspect vessel wall morphology, evaluate for the presence of plaques or thrombi and select appropriate landing zones for endografts (14). A particularly important application of IVUS is confirmation of the true vessel lumen and 


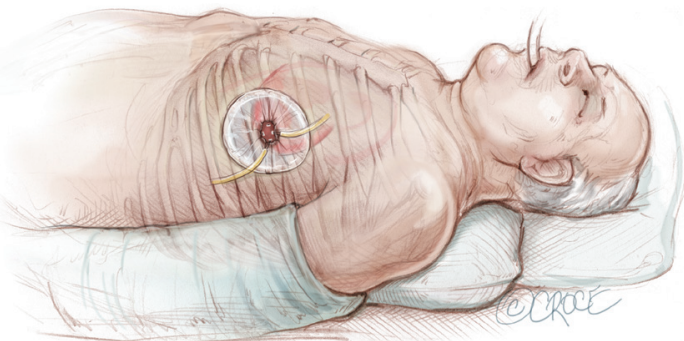

Figure 3 Anterior thoracotomy incision in $5^{\text {th }}$ or $6^{\text {th }}$ intercostal space with a chest wall soft tissue retractor placed for enhanced exposure.

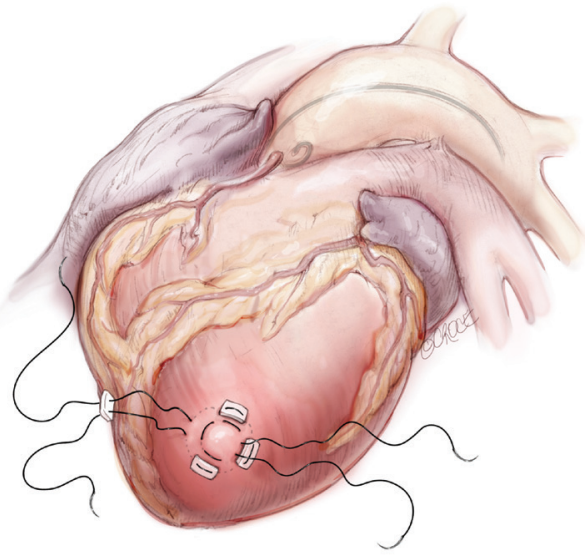

Figure 4 Two concentric purse-string sutures with 2-0 polypropylene on a $\mathrm{MH}$ needle are placed into the myocardium of the left ventricular (LV) apex.

localization of intimal tears in aortic dissection. After deployment of the endograft, IVUS permits confirmation of adequate stent graft position, complete apposition of the device to the aortic wall and evaluation for endoleaks. It also allows for assessment of true and false lumen diameter and flow to the branch vessels with aortic dissection. IVUS can significantly reduce the amount of contrast material used and exposure to fluoroscopy for both the patient and the team of healthcare providers.

\section{Endovascular technique}

Retrograde access to the ascending aorta via the femoral vessels can be utilized if the ilio-femoral vessels are of adequate size to accommodate the delivery sheaths of the stent grafts. Alternatively, the stent graft can be delivered through the left ventricular apex. The transapical access has

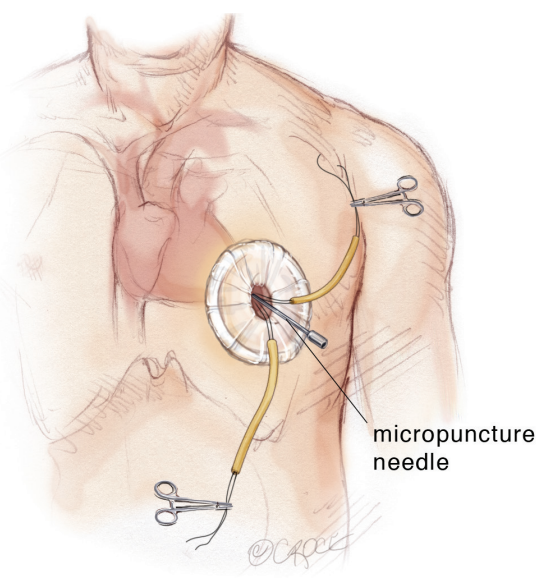

Figure 5 A micropuncture needle is used to gain entry into the left ventricular $(\mathrm{LV})$ cavity with the needle directed toward the right shoulder. Transesophageal echocardiography (TEE) is used to confirm positioning of the needle in the LV apex.

the advantages of its direct approach to the target area and that it avoids passage of the delivery system through aortic arch, it may therefore minimize embolic complications or extension of the aortic dissection resulting from arch manipulation (Figure 3). This approach may also facilitate entry into the true lumen and avoids a challenging delivery through anatomically unsuitable peripheral access vessels.

The transapical left ventricular approach for ascending TEVAR is performed through a small thoracotomy incision through the $5^{\text {th }}$ or $6^{\text {th }}$ intercostal space (Figure 3). The position of the $L V$ apex and determination of the level of incision is aided by preoperative CT imaging. Fluoroscopy is used to confirm alignment of the interspace with the apex of the left ventricle. The pericardium is incised and intraoperative TEE is used to confirm the location of the LV apex. Two concentric purse-string sutures with 2-0 polypropylene on a $\mathrm{MH}$ needle are placed into the myocardium of the LV apex (Figure 4) and a micropuncture needle and guidewire are used to gain entry into the LV cavity (Figure 5). Positioning of the guidewire is confirmed with fluoroscopy and an 8-French sheath then advanced over a soft-tip J-wire into the LV outflow tract (Figure 6). The patient is administered heparin to achieve a target activated clotting time $\geq 250$ seconds. This level of anticoagulation is maintained throughout the procedure, until distal pulses are verified after completion of the procedure. A 5F balloon-tipped temporary transvenous bipolar pacemaker is advanced into the RV apex through a femoral venous sheath. 


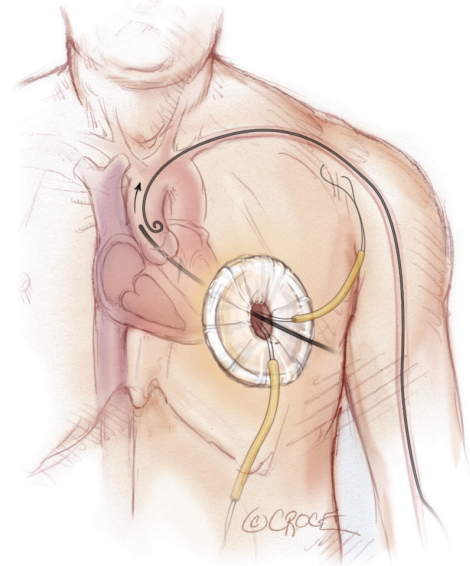

Figure 6 A pigtail catheter has been advanced into the ascending aorta via left radial access and a transapical sheath advanced through the aortic valve into the descending aorta.

The IVUS and guidewire are subsequently advanced together, under direct IVUS imaging and fluoroscopy, into the descending thoracic aorta. This is to ensure that the guidewire is in the true lumen. The guidewire is then exchanged through the IVUS catheter for a stiff (soft tip) guidewire and the ascending aorta interrogated with IVUS. The IVUS is used to examine the aortic root, ascending aorta and aortic arch as well as to identify the location of the intimal tear, the coronary arteries and the innominate artery. Appropriate diameter measurements of the proximal and distal landing zones above the sinotubular junction and proximal to the innominate artery are obtained as well as the distance from the coronary arteries to the innominate artery.

Additionally a 5-F pigtail catheter (transfemoral or through left brachial or radial access) is placed in the aortic root. It is used to perform an aortogram and evaluate the ascending aorta and aortic root anatomy including the location of the coronary arteries. The length and diameter of the proximal and distal landing zones can also be measured by this imaging modality. Using the preoperative CT scan, intraoperative IVUS as well as the angiogram, an appropriate stent graft is chosen. We recommend no greater than $10 \%$ oversizing relative to proximal landing zone diameter with aortic dissection or intramural hematoma. A delivery sheath is then advanced into the ascending aorta transapically through the aortic valve or via transfemoral access in a total endovascular approach (Figure 7). In a transfemoral approach, advancement of the delivery system into the aortic root requires the tip of the delivery system

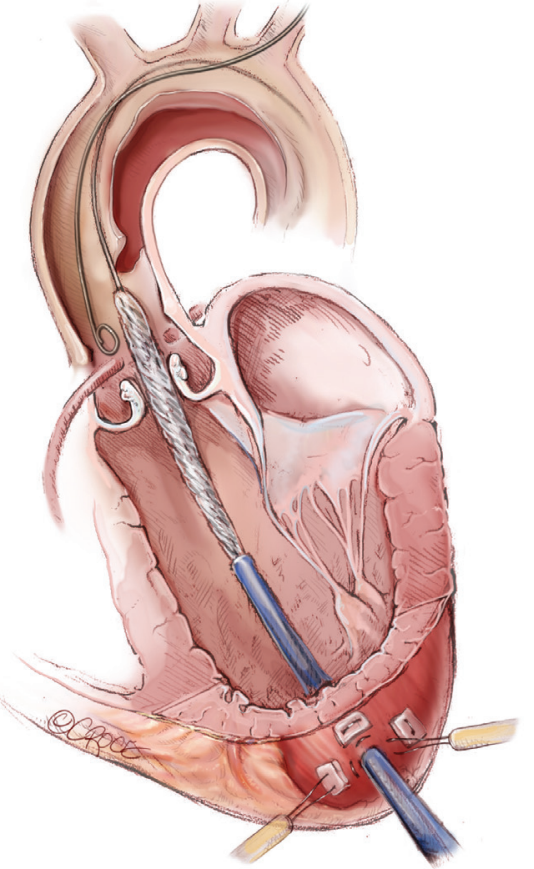

Figure 7 The ascending stent graft is advanced over the stiff guidewire through the valve into the true lumen of the dissection. A pigtail catheter is positioned in the aortic root to perform an aortogram immediately prior to stent graft deployment.

to be positioned through the aortic valve into the left ventricle. This poses a risk of injury to the aortic valve or left ventricle.

The stent graft position is targeted at least $5 \mathrm{~mm}$ above the coronary artery ostia and may partially cover the innominate artery at one third of the orifice or less if the transcranial Doppler reveals no significant change in velocities in the right internal carotid artery (Figure 8). In addition, aortic arch debranching with left common carotid to right common carotid bypass can be performed if complete coverage of the innominate artery is required to achieve an adequate distal seal zone. The aortic stent graft is deployed with rapid right ventricular pacing at a rate of 160 to 180 beats per minute or administration of adenosine $36 \mathrm{mg}$ intravenously to minimize cardiac output and ensure precise device deployment.

Intraoperative TEE confirms proximal positioning of the stent graft at or above the sinotubular junction, adequate function of the aortic valve and cardiac function and lack of a significant pericardial effusion. After completion angiography and IVUS assessment to confirm adequate device apposition to the adjacent aorta in the proximal and 


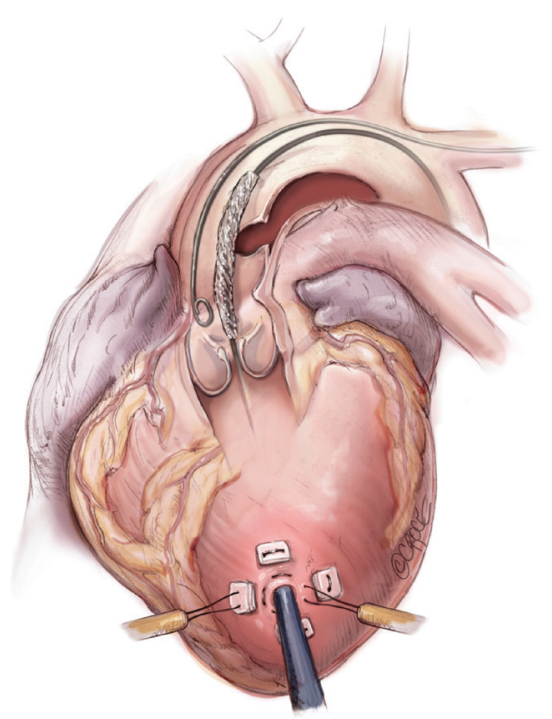

Figure 8 Stent graft delivery system being advanced into the aortic root in preparation for deployment.

distal seal zones (Figure 9) and patency of the coronary arteries, the introducer sheath is removed and the pursestring sutures in the LV apex tied with ventricular pacing at a rate of 120 beats per minute. In the transfemoral approach, an angiogram is usually performed after removal of the introducer sheath and an iliofemoral angiogram may be performed if there is concern of injury to the access vessels.

\section{Discussion}

The anatomic suitability for TEVAR in type A dissections has been investigated by Moon and colleagues (15). They reported that $32 \%$ of patients with acute type A dissections with CT scans of sufficient quality to allow assessment for endovascular treatment were anatomically suitable for endovascular repair. The absence of an acceptable proximal landing zone was the most common exclusion criterion for possible endovascular repair. Sobocinski et al. also reviewed CTs and clinical data from patients with Stanford type A aortic dissection for feasibility of endovascular repair (16). Additional debranching of the innominate trunk to the left common carotid artery was considered as acceptable to increase the length of the distal landing zone. Their study shows that approximately half of the patients may meet anatomic criteria determining eligibility for TEVAR.

There are few reports in the medical literature of successful treatment of acute type A aortic dissection

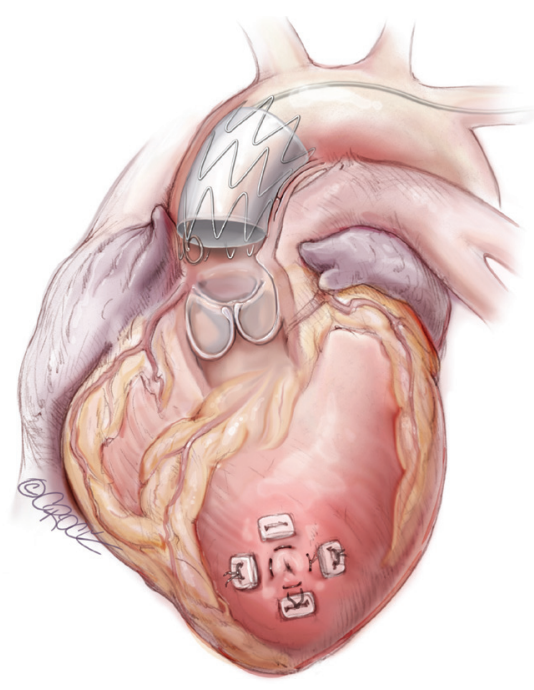

Figure 9 Completed transapical ascending stent graft deployment.

utilizing endovascular techniques. The first reported case of endovascular treatment of type A aortic dissection was by Dorros and colleagues in 2000 utilizing a transseptal technique (17). A guide wire was passed from the right femoral vein, across both atria, the left ventricle and then into the ascending aorta. Senay and colleagues reported successful endovascular stent graft treatment with simultaneous coronary stenting in a patient with type A aortic dissection and the primary entry tear located in the ascending aorta (18).

Recently, successful endovascular stenting of the ascending aorta in four patients with type A aortic dissection was reported by Ronchey and colleagues using a transfemoral approach (19). The anatomic inclusion criteria were the following: intimal entry tear location in the ascending aorta at least $2 \mathrm{~cm}$ above the sinotubular junction and at least $0.5 \mathrm{~cm}$ proximal to the innominate artery as well as no evidence of severe aortic valve insufficiency, cardiac tamponade, aortic branch malperfusion or patent coronary artery bypass grafts. They reported a $100 \%$ technical success rate and no mortality or migration of the stent grafts after a median follow-up of 15 months. In one patient, a bypass was performed from the left common carotid artery (LCC) to the right common carotid artery (RCC) and to the right subclavian artery to extend the distal landing zone.

Ye et al. published in 2011 a large series of 45 patients with type A aortic dissection treated with TEVAR (20). The surgical success rate was $97 \%$ and the 30 -day mortality rate $6.7 \%$. The entry tear was located in the ascending aorta in 10 cases, in the aortic arch in 14 cases and in the proximal 
descending aorta in 21 cases. For the first two patients in whom the tear was in the ascending aorta, the stent graft was inserted through the left common carotid (LCC) artery due to the rigidity of the first generation stents. Prior to this delivery, a bypass was sutured from the left subclavian artery (LSA) to LCC and ensured cerebral perfusion. These two patients suffered stroke 3 weeks and 1 year postoperatively. With the development of stent grafts that facilitate the passage through the aortic arch, the approach was switched to transfemoral. Endoleak type 1 occurred in 10 of 45 cases and was initially treated with balloon dilatation in the landing zone, avoiding the area of the entry tear or dilatation of the whole stent. If the endoleak persisted, an aortic extending cuff was used to seal this tear.

$\mathrm{Lu}$ and associates reported a series of 15 patients with ascending aortic dissection who were deemed unfit for open surgical repair and who underwent endovascular repair from 2009 to 2011 (21). The median time from onset of dissection to treatment was 25.5 days, with 5 patients considered to be acute who underwent treatment within 14 days. Exclusion criteria for aortic stent grafting were: proximal or distal landing zone $<20 \mathrm{~mm}$, involvement of coronary ostia, acute myocardial infarction, grade 3 or 4 aortic regurgitation, severe tortuosity of the descending aorta or connective tissue disorder. Technical success was reported in $100 \%$, with no perioperative deaths or strokes reported in this series. One patient developed a new dissection in the aortic arch 3 months after ascending TEVAR, which was treated with a branched endograft.

TEVAR for acute type A dissection may be associated with a spectrum of complications. The most serious complications of TEVAR are neurologic resulting from ischemia or embolization. Perioperative acute ischemic stroke associated with TEVAR has a reported incidence of $3.5 \%$ to $5.5 \%(22,23)$ and is more common if the TEVAR involves more proximal aorta (landing zones 0-2) (24). Excessive wire and catheter manipulation in a diseased aortic arch with risks of air embolism from deployment systems and inadvertent coverage of arch branches, increases the risk of stroke. The passage of a delivery system through aortic arch may cause neuroembolic complications or extension of the dissection. The use of transcranial Doppler ultrasound and intravascular ultrasound as well as proper selection of catheters, wires and maneuvers help to diagnose and may reduce the risk of neuro-embolization (25).

The proximity of the coronary artery ostia and aortic valve to the proximal landing zone increases the risk of coronary artery coverage or aortic valve dysfunction. The fragility of the ascending aorta in the setting of acute dissection increases the risk of aortic rupture or retrograde dissection during stent graft deployment. Another risk after TEVAR is the presence of endoleaks, characterized by the persistence of blood flow within the aneurysm sac. The incidence of endoleaks following TEVAR in a recent metaanalysis of reported studies is $12.1 \%$ (26). This represents a particular challenge in the ascending aorta. These may be treated either with additional balloon dilatation or an additional stent graft to extend the proximal or distal landing zone.

\section{Conclusions}

The current endovascular stent graft technology offers an alternative treatment option in selected high-risk patients with acute type A dissection who are unfit for surgical repair. It is built upon current TEVAR and TAVR technology. Since there are still many technical issues that need to be resolved, future innovations will provide more disease-specific devices and solutions to support physicians in expanding the indications for TEVAR.

\section{Acknowledgements}

None.

\section{Footnote}

Conflicts of Interest: The authors have no conflicts of interest to declare.

\section{References}

1. Suzuki T, Mehta RH, Ince H, et al. Clinical profiles and outcomes of acute type $\mathrm{B}$ aortic dissection in the current era: lessons from the International Registry of Aortic Dissection (IRAD). Circulation 2003;108 Suppl 1:II312-7.

2. Olsson C, Thelin S, Ståhle E, et al. Thoracic aortic aneurysm and dissection: increasing prevalence and improved outcomes reported in a nationwide populationbased study of more than 14,000 cases from 1987 to 2002 . Circulation 2006;114:2611-8.

3. Svensson LG, Kouchoukos NT, Miller DC, et al. Expert consensus document on the treatment of descending thoracic aortic disease using endovascular stent-grafts. Ann Thorac Surg 2008;85:S1-41.

4. Nienaber CA, Eagle KA. Aortic dissection: new frontiers 
in diagnosis and management: Part II: therapeutic management and follow-up. Circulation 2003;108:772-8.

5. Pape LA, Awais M, Woznicki EM, et al. Presentation, Diagnosis, and Outcomes of Acute Aortic Dissection: $17-$ Year Trends From the International Registry of Acute Aortic Dissection. J Am Coll Cardiol 2015;66:350-8.

6. Hirst AE Jr, Johns VJ Jr, Kime SW Jr. Dissecting aneurysm of the aorta: a review of 505 cases. Medicine (Baltimore) 1958;37:217-79.

7. DeBakey ME, Henley WS, Cooley DA, et al. Surgical treatment of dissecting aneurysm of the aorta. J Thorac Cardiovasc Surg 1965;49:130-49.

8. Dake MD, Thompson M, van Sambeek M, et al. DISSECT: a new mnemonic-based approach to the categorization of aortic dissection. Eur J Vasc Endovasc Surg 2013;46:175-90.

9. Eggebrecht H, Thompson M, Rousseau H, et al. Retrograde ascending aortic dissection during or after thoracic aortic stent graft placement: insight from the European registry on endovascular aortic repair complications. Circulation 2009;120:S276-81.

10. Fattori R, Lovato L, Buttazzi K, et al. Extension of dissection in stent-graft treatment of type B aortic dissection: lessons learned from endovascular experience. J Endovasc Ther 2005;12:306-11.

11. Neuhauser B, Czermak BV, Fish J, et al. Type A dissection following endovascular thoracic aortic stent-graft repair. J Endovasc Ther 2005;12:74-81.

12. van Prehn J, Vincken KL, Muhs BE, et al. Toward endografting of the ascending aorta: insight into dynamics using dynamic cine-CTA. J Endovasc Ther 2007;14:551-60.

13. Kobeiter H, Nahum J, Becquemin JP. Zero-contrast thoracic endovascular aortic repair using image fusion. Circulation 2011;124:e280-2.

14. White RA, Donayre CE, Walot I, et al. Intraprocedural imaging: thoracic aortography techniques, intravascular ultrasound, and special equipment. J Vasc Surg 2006;43 Suppl A:53A-61A.

15. Moon MC, Greenberg RK, Morales JP, et al. Computed tomography-based anatomic characterization of proximal aortic dissection with consideration for endovascular

Cite this article as: Shah A, Khoynezhad A. Thoracic endovascular repair for acute type A aortic dissection: operative technique. Ann Cardiothorac Surg 2016;5(4):389-396. doi: 10.21037/acs.2016.07.08 candidacy. J Vasc Surg 2011;53:942-9.

16. Sobocinski J, O'Brien N, Maurel B, et al. Endovascular approaches to acute aortic type A dissection: a CT-based feasibility study. Eur J Vasc Endovasc Surg 2011;42:442-7.

17. Dorros G, Dorros AM, Planton S, et al. Transseptal guidewire stabilization facilitates stent-graft deployment for persistent proximal ascending aortic dissection. J Endovasc Ther 2000; 7:506-12.

18. Senay S, Alhan C, Toraman F, et al. Endovascular stentgraft treatment of type A dissection: case report and review of literature. Eur J Vasc Endovasc Surg 2007;34:457-60.

19. Ronchey S, Serrao E, Alberti V, et al. Endovascular stenting of the ascending aorta for type A aortic dissections in patients at high risk for open surgery. Eur J Vasc Endovasc Surg 2013;45:475-80.

20. Ye C, Chang G, Li S, et al. Endovascular stent-graft treatment for Stanford type A aortic dissection. Eur J Vasc Endovasc Surg 2011;42:787-94.

21. Lu Q, Feng J, Zhou J, et al. Endovascular repair of ascending aortic dissection: a novel treatment option for patients judged unfit for direct surgical repair. J Am Coll Cardiol 2013;61:1917-24.

22. Khoynezhad A, Donayre CE, Bui H, et al. Risk factors of neurologic deficit after thoracic aortic endografting. Ann Thorac Surg 2007;83:S882-9; discussion S890-2.

23. Ehlert BA, Durham CA, Parker FM, et al. Impact of operative indication and surgical complexity on outcomes after thoracic endovascular aortic repair at National Surgical Quality Improvement Program Centers. J Vasc Surg 2011;54:1629-36.

24. Melissano G, Tshomba Y, Bertoglio L, et al. Analysis of stroke after TEVAR involving the aortic arch. Eur J Vasc Endovasc Surg 2012;43:269-75.

25. Khoynezhad A, Celis R. Transcranial Doppler-guided selective antegrade cerebral perfusion during aortic debranching operation. J Thorac Cardiovasc Surg 2009;138:1029-30.

26. Cheng D, Martin J, Shennib H, et al. Endovascular aortic repair versus open surgical repair for descending thoracic aortic disease a systematic review and meta-analysis of comparative studies. J Am Coll Cardiol 2010;5 5:986-1001. 